\title{
\begin{tabular}{l|l} 
Mitraries & DSpace@MIT
\end{tabular}
}

\author{
MIT Open Access Articles
}

\section{An approach for incorporating classical continuum damage models in state-based peridynamics}

The MIT Faculty has made this article openly available. Please share how this access benefits you. Your story matters.

Citation: Tupek, M.R.; Rimoli, J.J. and Radovitzky, R. “An Approach for Incorporating Classical Continuum Damage Models in State-Based Peridynamics." Computer Methods in Applied Mechanics and Engineering 263 (August 2013): 20-26. (c) 2013 Elsevier B.V.

As Published: http://dx.doi.org/10.1016/j.cma.2013.04.012

Publisher: Elsevier

Persistent URL: http://hdl.handle.net/1721.1/108095

Version: Author's final manuscript: final author's manuscript post peer review, without publisher's formatting or copy editing

Terms of use: Creative Commons Attribution-NonCommercial-NoDerivs License 


\title{
An approach for incorporating classical continuum damage models in state-based peridynamics
}

\author{
M.R. Tupek ${ }^{\mathrm{a}}$, J.J. Rimolia,b, R. Radovitzky ${ }^{\mathrm{a}, *}$ \\ ${ }^{a}$ Department of Aeronautics and Astronautics \\ Massachusetts Institute of Technology \\ Cambridge, MA 02139 \\ ${ }^{b}$ School of Aerospace Engineering \\ Georgia Institute of Technology \\ Atlanta, GA 30332
}

\begin{abstract}
Peridynamics has gained significant attention as an alternative formulation for problems in solid mechanics. Recent contributions have included initial attempts to include material damage and failure. In this paper, we propose an approach to incorporate classical continuum damage models in the state-based theory of peridynamics. This has the advantage of enabling the description of the damage evolution process in peridynamics according to well-established models. The approach is based on modifying the peridynamic influence function according to the state of accumulated damage. As a result, peridynamic bonds between nonlocal material points are severed in accordance with the damage law. The peridynamic damage formulation proposed is implemented for the particular case of a well established ductile damage model for metals. The model is applied to the simulation of ballistic impact of extruded corrugated aluminum panels and compared with experiments.
\end{abstract}

Keywords: peridynamics, damage modeling, particle methods

\footnotetext{
* Corresponding author

Email address: rapa@mit.edu (R. Radovitzky)
} 


\section{Introduction}

Peridynamics is a non-local continuum theory of solid mechanics originally proposed by Silling to address elasticity problems involving discontinuities and long-range forces [1]. One of its main objectives is to provide a formulation which naturally supports the presence of discontinuities in the deformation field. Another advantage of the theory is that the resulting equations of motion are naturally discretized using particle-based methods [2]. This is presumed to have advantages in problems involving severe material deformations where meshbased discretizations fail. One of the key remaining challenges in peridynamics and associated discretization methods is how to describe material failure in ways which are consistent with established models of fracture and damage.

In the original formulation of peridynamics, usually referred to as the bondbased theory, fracture is commonly incorporated by means of a critical relative displacement criterion, i.e., when the change in distance between two particles reaches a critical value $u_{c}$, their bond is irreversibly broken [3]. A particle-based discretization of peridynamic was proposed in [4], where it was demonstrated that the critical bond elongation $u_{c}$ can be related to the fracture energy $G_{0}$ for brittle materials. This approach has been used for modeling fracture and failure of composites, nanofiber networks and polycrystals [5], to simulate ballistic impact on brittle plates [6], to study crack nucleation in peridynamic solids [7], and to study dynamic crack propagation and crack branching [8]. The main limitation of the bond-based peridynamic theory is that it only considers pairwise interactions between particles. As is well known, a direct consequence of this assumption is that the effective Poisson's ratio for isotropic linear materials is fixed at the value of $\nu=0.25$ [1]. An immediate repercussion of this limitation is the inadequacy of the bond-based peridynamics formulation in situations involving incompressible deformations, e.g. plasticity. 
To address these issues, Silling et al. developed the so called state-based peridynamics formulation [9], which makes it possible to incorporate general constitutive models. In particular, the new formulation introduces a constitutive correspondence framework which enables the use of traditional constitutive models formulated in terms of a continuum local measure of deformation (i.e., the deformation gradient tensor, F). Recently, this approach was used to model viscoplastic deformations in metals $[10,2]$. The ability to incorporate classical constitutive models also opens the path for using classical continuum damage models within the peridynamics framework.

Existing state-based peridynamic damage modeling approaches in the literature are based on permanently modifying the peridynamic influence function by instantly setting it equal to zero and severing the bond when a failure criterion is achieved $[10,11]$. Within the context of ordinary state-based peridynamics, the role of the influence function has been explored in [12], and a critical bond elongation criterion has been proposed in [11] which is similar to the damage modeling approach commonly used in bond-based peridynamics $[3,4,5,6,7,8]$. A severing criterion based on a maximum elastic bond energy was proposed in [10] for the constitutive correspondence formulation and calibrated to dissipate a pre-specified fracture energy when a new surface is created. These approaches appear to be successful for modeling brittle fracture. However, there are situations (e.g. in ductile fracture), in which damage evolution and failure are known to depend on quantities such as the stress triaxiality, Lode angle, and possibly other parameters characterizing the local stress state $[13,14,15]$. It would therefore be desirable to be able to incoporate classical damage models whose primary objective is the description of damage mechanisms and their evolution in a physics-based or phenomenological manner.

A more general framework for modeling damage within peridynamics has 
recently been proposed [16]. The theory constitutes a thermodynamically consistent extension of state-based peridynamics where accumulated damage is represented by a damage-state. However, the requirement to introduce a peridynamic damage-state makes it impossible to use existing damage models directly, and the necessary reformulation within this framework has yet to be done for general damage models. Specifically, it is not clear how the Johnson-Cook damage model adopted in the examples in this paper should be modified to fit this general framework.

The main objective of this paper is to develop a state-based peridynamics formulation where classical (local) continuum damage models can be incorporated without modification. It is found that a direct implementation of damage models within the constitutive correspondence framework leads to instabilities associated with unphysical diffusion of the damage zone. To address this issue, we employ a peridynamic bond degradation criterion based on the accumulated material damage. As damage evolves at a material point, the peridynamic influence function for bonds in the neighborhood is decreased so that in the limit of full damage its interaction with other material points vanishes. This can be viewed as an extension of previous bond-severing criteria in state-based peridynamics to more general cases where the influence function is allowed to degrade gradually and to have a general dependence on other state variables such as plastic strain, void volume fraction, temperature, etc., thereby enabling the description of the damage evolution process. To assess the method, we consider the specific case of the Johnson-Cook plasticity and damage model [17].

In Section 2, we present the state-based peridynamic damage formulation and discuss the implementation of the method for the specific damage model considered. In Section 3, we present numerical results consisting of: 1) the simulation of a Taylor impact test which is used for verification against other numer- 
ical methods; and 2) the simulation of ballistic impact of extruded aluminum sandwich panels by steel spheres. A summary and discussion with suggestions for future research is presented in Section 4 .

\section{Model description}

For completeness, we briefly review the state-based peridynamic theory including a summary of the peridynamic equations of motion, and the idea of constitutive correspondence and its key properties. Subsequently, we describe the modified theory to incorporate constitutive damage and demonstrate the integration of a specific classical damage model into the peridynamic theory. We conclude this section with some details about the numerical implementation.

\subsection{Peridynamic states}

State-based peridynamics is a non-local continuum theory describing the dynamics of a peridynamic body which we assume occupies the region $\mathcal{B}_{0} \subset \mathbb{R}^{3}$ in the reference configuration at time $t=0$ and the region $\mathcal{B}_{t} \subset \mathbb{R}^{3}$ at time

$t$. Consider material points in the reference configuration $\mathbf{x}, \mathbf{x}^{\prime} \in \mathcal{B}_{0}$. Under the deformation mapping $\varphi: \mathcal{B}_{0} \rightarrow \mathcal{B}_{t}$, points $\mathbf{x}$ and $\mathbf{x}^{\prime}$ map to $\mathbf{y}$ and $\mathbf{y}^{\prime}$ respectively. From the perspective of point $\mathbf{x}$, the bond to $\mathbf{x}^{\prime}$ is the vector

$$
\xi=\mathbf{x}^{\prime}-\mathbf{x}
$$

Using similar definitions to those provided in [16], we define a family $\mathcal{H}$ at $\mathbf{x}$ by

$$
\mathcal{H}(\mathbf{x})=\left\{\boldsymbol{\xi} \in \mathbb{R}^{3}\left|(\boldsymbol{\xi}+\mathbf{x}) \in \mathcal{B}_{0},\right| \boldsymbol{\xi} \mid<\delta\right\}
$$

where $\delta>0$ is the horizon radius, which defines a physical length scale in the continuum formulation. A schematic of a peridynamic body and the family at $\mathbf{x}$ is shown in Figure 1. 


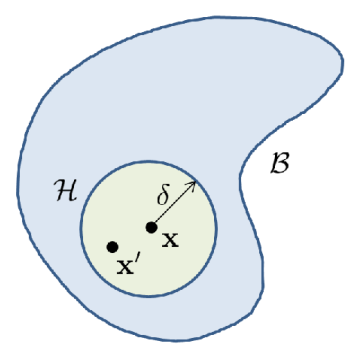

Figure 1: Schematic representation of a body $\mathcal{B}$ and the family $\mathcal{H}$ of the point $\mathbf{x}$.

A peridynamic vector-state $\underline{\mathbf{a}}[\mathbf{x}]$ at a point $\mathbf{x} \in \mathcal{B}_{0}$ is a function

$$
\underline{\mathbf{a}}[\mathbf{x}]\langle\cdot\rangle: \mathcal{H}(\mathbf{x}) \rightarrow \mathbb{R}^{3} .
$$

An important example of a vector-state is the deformation vector-state $\underline{\mathbf{Y}}$, which is defined at $\mathbf{x}$ by

$$
\underline{\mathbf{Y}}\langle\boldsymbol{\xi}\rangle \equiv \mathbf{y}^{\prime}-\mathbf{y} .
$$

Vector-states take an analogous role in state-based peridynamic theory as tensors do in classical local continuum mechanics. For example, the deformation vector-state is analogous to the deformation gradient in classical theories, but is able to describe more general kinematics [16].

What differentiates peridynamics from classical continuum mechanics in an essential way is that the theory is inherently non-local in the sense that material points are assumed to interact through long-range forces represented by the force vector-state $\underline{\mathbf{T}}$, where $\underline{\mathbf{T}}\langle\boldsymbol{\xi}\rangle \in \mathbb{R}^{3}$ can be loosly interpreted as a force density per unit reference volume at point $\mathbf{x}$ due to interactions with the point $\mathbf{x}^{\prime}$.

This state-based peridynamic formulation is a generalization of the bondbased peridynamic framework which allows the response of the material at a given point to depend on the collective deformation of all the bonds connected 
to that point [9]. The peridynamic equations of motion are given by

$$
\rho(\mathbf{x}) \ddot{\mathbf{y}}(\mathbf{x}, t)=\int_{\mathcal{H}(\mathbf{x})}\{\underline{\mathbf{T}}[\mathbf{x}, t]\langle\boldsymbol{\xi}\rangle-\underline{\mathbf{T}}[\mathbf{x}+\boldsymbol{\xi}, t]\langle-\boldsymbol{\xi}\rangle\} d V_{\boldsymbol{\xi}}+\mathbf{b}(\mathbf{x}, t),
$$

where $\rho$ is the density of the material and $\mathbf{b}$ is an externally applied body force. The peridynamic equations of motion enforce conservation of linear momentum. In the following, the more concise "prime" notation: $\underline{\mathbf{T}}\langle\boldsymbol{\xi}\rangle \equiv \underline{\mathbf{T}}[\mathbf{x}, t]\left\langle\mathrm{x}^{\prime}-\mathbf{x}\right\rangle$, and $\underline{\mathbf{T}}^{\prime}\langle-\boldsymbol{\xi}\rangle \equiv \underline{\mathbf{T}}\left[\mathbf{x}^{\prime}, t\right]\left\langle\mathbf{x}-\mathbf{x}^{\prime}\right\rangle$ will be used. The equations of motion in this notation are then

$$
\rho \ddot{\mathbf{y}}=\int_{\mathcal{H}} \underline{\mathbf{T}}\langle\boldsymbol{\xi}\rangle-\underline{\mathbf{T}}^{\prime}\langle-\boldsymbol{\xi}\rangle d V_{\boldsymbol{\xi}}+\mathbf{b}
$$

where the dependence on $\mathbf{x}$ and $t$ is implied.

A key assumption of the state-based peridynamic theory is that the force vector-state $\underline{\mathbf{T}}$ at each material point $\mathbf{x}$ is a function of the deformation vectorstate $\underline{\mathbf{Y}}$ defined in equation (1), and possibly of the rate of deformation vectorstate $\underline{\dot{\mathbf{Y}}}$, temperature $\theta$, and other internal variables $\mathbf{q}$ as well. Restrictions on the form of $\underline{\mathbf{T}}$ are required to ensure conservation of angular momentum and to satisfy the first and second laws of thermodynamics [16].

In order to facilitate the incorporation of classic constitutive models into the peridynamic framework, Silling et al. [9] introduced the concept of peridynamic constitutive correspondence. A peridynamic material model is said to correspond to a classical material model when the strain energy density of both the classical and peridynamic material are equal under affine deformations. For this purpose, an approximate deformation gradient field $\overline{\mathbf{F}}$ was introduced:

$$
\overline{\mathbf{F}}(\underline{\mathbf{Y}})=\left[\int_{\mathcal{H}} \omega(|\boldsymbol{\xi}|)(\underline{\mathbf{Y}}\langle\boldsymbol{\xi}\rangle \otimes \boldsymbol{\xi}) d V_{\boldsymbol{\xi}}\right] \overline{\mathbf{K}}^{-1}
$$


where $\omega(|\boldsymbol{\xi}|)$ is a scalar valued radial influence function which depends only on the length of the bond $\boldsymbol{\xi}$, and $\overline{\mathbf{K}}$ is a non-local shape tensor defined by

$$
\overline{\mathbf{K}}=\int_{\mathcal{H}} \omega(|\boldsymbol{\xi}|)(\boldsymbol{\xi} \otimes \boldsymbol{\xi}) d V_{\boldsymbol{\xi}}
$$

Alternatively, we can define this integral without recourse to peridynamics states as follows:

$$
\begin{gathered}
\overline{\mathbf{F}}(\mathbf{x}, t)=\int_{\Omega_{x}} \omega\left(\left|\mathbf{x}^{\prime}-\mathbf{x}\right|\right)\left[\left(\mathbf{y}\left(\mathbf{x}^{\prime}, t\right)-\mathbf{y}(\mathbf{x}, t)\right) \otimes\left(\mathbf{x}^{\prime}-\mathbf{x}\right)\right] d V_{\mathbf{x}^{\prime}} \overline{\mathbf{K}}^{-1}(\mathbf{x}), \\
\overline{\mathbf{K}}(\mathbf{x})=\int_{\Omega_{x}} \omega\left(\left|\mathbf{x}^{\prime}-\mathbf{x}\right|\right)\left[\left(\mathbf{x}^{\prime}-\mathbf{x}\right) \otimes\left(\mathbf{x}^{\prime}-\mathbf{x}\right)\right] d V_{\mathbf{x}^{\prime}},
\end{gathered}
$$

where $\Omega_{x}=\left\{\mathbf{x}^{\prime} \in \mathcal{B}_{0}|| \mathbf{x}^{\prime}-\mathbf{x} \mid<\delta\right\}$. It can be seen from equation 4 that the constitutive correspondence form of $\overline{\mathbf{F}}$ provides a description of the state of deformation at point $\mathbf{x}$, based on a weighted average of the deformation of all the neighboring bonds. It has been shown that the approximate deformation gradient obtained in this way is identical to the classic deformation gradient (i.e., the continuous gradient of the deformation mapping, F) for affine deformations $[9]$.

The force vector-state for constitutive correspondence takes the form:

$$
\underline{\mathbf{T}}\langle\boldsymbol{\xi}\rangle=\omega(|\boldsymbol{\xi}|) \overline{\mathbf{P}} \overline{\mathbf{K}}^{-1} \boldsymbol{\xi},
$$

where $\overline{\mathbf{P}}=\hat{\mathbf{P}}(\overline{\mathbf{F}})$ is the first Piola-Kirchhoff stress tensor obtained from a classic constitutive law as a function of the approximate deformation gradient. This form of the force vector-state leads to a peridynamic formulation which conserves angular momentum provided the classical constitutive model used is one which ensures conservation of angular momentum and is non-polar. This is the case if 
the resulting Cauchy stress $\overline{\boldsymbol{\sigma}}$ is symmetric, i.e., $\overline{\boldsymbol{\sigma}}=\bar{J}^{-1} \overline{\mathbf{P}} \overline{\mathbf{F}}^{T}=\overline{\boldsymbol{\sigma}}^{T}$.

\subsection{Damage correspondence}

The ability to incorporate existing classical constitutive models provided by the correspondence formulation opens up the possibility of using classical continuum descriptions of damage within the peridynamic framework. However, it can be easily seen that an inattentive use of such models results in unphysical instabilities in numerical computations. Specifically, when a material particle in the peridynamic body is fully damaged, the material is able to flow unconstrained. Due to the non-local character of the peridynamic deformation gradient $\overline{\mathbf{F}}$, material points within the horizon of fully damaged regions will then compute unphysically-large strains which, in turn, will lead to unphysical damage. As a result, there is a tendency for damage to diffuse in an unrealistic manner in situations where damage should in fact localize into regions whose width should be of the order of the horizon size. It is therefore necessary to augment the peridynamic formulation to preserve the ability for damage to localize.

Instabilities in peridynamic simulations have been previously observed in the form of zero energy modes [18], which are common to many particle based continuum discretizations $[19,20]$. These zero energy modes can manifest themselves even in purely elastic simulations. However, the instability we focus on here appears to be primarily due to unphysical propagation of damage.

In order to address this issue, we adopt a generalized definition of the influence function $\omega$, equation (3), and make it dependent not only on the length of the bond $|\boldsymbol{\xi}|$, but also on the internal state-variables $\left(\mathbf{q}, \mathbf{q}^{\prime}\right)$ :

$$
\omega=\hat{\omega}\left(|\boldsymbol{\xi}|, \mathbf{q}, \mathbf{q}^{\prime}\right)
$$


where $\mathbf{q}$ and $\mathbf{q}^{\prime}$ are vectors of internal or state variables at the material points $\mathbf{x}$ and $\mathbf{x}^{\prime}$ respectively. This strategy can be viewed as an extension of previous approaches to state-based peridynamic damage modeling in which the influence function is set to zero when a bond failure criterion is satisfied $[10,11,12]$ with two major differences: 1) the proposed formulation contemplates the possibility that the influence function may depend smoothly on the internal fields, resulting in a gradual degradation of material point interactions. In addition, 2) both the evolution of the damage process and the ultimate material failure criterion are governed by the classical continuum damage model which in general may depend on any of the internal state fields (e.g. plastic strain, void volume fraction, temperature, stress triaxiality, Lode angle, as well as material properties). These two considerations are probably critical to fully capture the damage mechanisms that are phenomenologically implied by the original continuum damage model.

In this paper we will assume for simplicity that the state of material damage in the constitutive model is described by a single parameter $D$, which is commonly the case in some of the most popular damage models used in practice. We consider influence functions of the form

$$
\hat{\omega}\left(|\boldsymbol{\xi}|, \mathbf{q}, \mathbf{q}^{\prime}\right)=\omega_{\xi}(|\boldsymbol{\xi}|) \omega_{D}\left(D, D^{\prime}\right)
$$

where $D$ and $D^{\prime} \in[0,1]$ are the values of the damage parameter at $\mathbf{x}$ and $\mathbf{x}^{\prime}$, respectively, and $\omega_{\xi}(|\boldsymbol{\xi}|)$ is the conventional (radial) influence function which quantifies the relative degree of interaction between neighboring material points in an undamaged material. We require $\omega_{D}$ to be a non-increasing function of each of its arguments and to be zero if either argument is 1, i.e., $\omega_{D}(a, b)=$ 0 if $a=1$ or $b=1$. In this way, the contribution of a damaged material point to the peridynamic force-state of neighboring points decreases as damage accumulates, and vanishes altogether when the material point is fully damaged. 
Any subsequent compressive response resulting from contact interactions are handled by a particle contact algorithm.

The specific forms we choose for the influence functions are

$$
\begin{gathered}
\omega_{\xi}(|\boldsymbol{\xi}|)=e^{-\frac{|\xi|^{2}}{\sigma^{2}}}, \\
\omega_{D}\left(D, D^{\prime}\right)= \begin{cases}0 & \text { if } D>D_{c}, \text { or } D^{\prime}>D_{c} \\
1 & \text { otherwise, }\end{cases}
\end{gathered}
$$

where $\sigma$ defines the physical length scale, and $D_{c}$ is the critical bond damage. A Gaussian influence function such as $\omega_{\xi}$ has previously been used in the context of bond-based peridynamics [21]. The influence function, equation (6), leads to equations of motion which conserve angular momentum, as can be shown by arguments identical to those presented in [9]. The only difference is the added explicit dependence on additional internal state-variables, $\mathbf{q}$ and $\mathbf{q}^{\prime}$, which does not affect momentum conservation.

\subsection{Constitutive model}

The damage correspondence formulation of Section 2.2 is general and can in principle be applied to a wide array of continuum damage models. For definiteness, we specialize it to a modified Johnson-Cook constitutive model of viscoplasticity and failure of metals $[22,17]$. In this model, the flow stress $Y$ is defined as

$$
Y=\sigma_{0}\left[1+\frac{\epsilon^{p}}{\epsilon_{0}^{p}}\right]^{n}\left[1+C \log \left(\frac{\dot{\epsilon}^{p}}{2 \dot{\epsilon}_{0}^{p}}+\sqrt{1+\left(\frac{\dot{\epsilon}^{p}}{2 \dot{\epsilon}_{0}^{p}}\right)^{2}}\right)\right]
$$


where $\epsilon^{p}$ is the equivalent plastic strain, $\dot{\epsilon}^{p}$ is the equivalent plastic strain rate,

$$
\epsilon_{0}^{p}=\left(\frac{\sigma_{0}}{B}\right)^{\frac{1}{n}}
$$

is a reference plastic strain, and $\dot{\epsilon}_{0}^{p}$ is a reference plastic strain rate. Compared to the classical Johnson-Cook flow stress, this modified expression avoids singularities in the hardening as $\dot{\epsilon}^{p} \rightarrow 0$ and in the hardening modulus as $\epsilon^{p} \rightarrow 0$. $[22,23,24,25]$. The elastic response is described using a hyperelastic formulations based on logarithmic strains and standard isotropic elastic constants.

As in the standard Johnson-Cook damage model [17], the evolution of damage is controled by the damage parameter given by

$$
\begin{gathered}
D=\int_{0}^{t} \dot{D} d \tau, \\
\dot{D}= \begin{cases}\frac{\dot{c}^{p}}{\epsilon_{f}} & \text { if } D<1 \\
0 & \text { otherwise }\end{cases}
\end{gathered}
$$

with $\epsilon_{f}$ defined as

$$
\epsilon_{f}=\left[d_{1}+d_{2} \exp \left(-d_{3} \frac{\sigma_{m}}{\sigma_{e}}\right)\right]\left[1+d_{4} \ln \left(\frac{\dot{\epsilon}^{p}}{\dot{\epsilon}_{0}}\right)\right],
$$

where $d_{1}, d_{2}, d_{3}, d_{4}$, and $\dot{\epsilon}_{0}$ are material parameters, $\sigma_{m}$ is the hydrostatic stress, and $\sigma_{e}$ is the Von Mises equivalent stress.

\subsection{Discretization}

The discretization approach adopted here follows [2]. The initial configuration of the problem domain is discretized into a set of $N$ particles which represent the volume of the Voronoi cells. Displacement degrees of freedom and 
other field and internal variable data are associated with each particle, which in effect implies a piecewise-constant interpolation supported on the Voronoi diagram. Particles located at $\mathbf{x}_{i} \in \mathcal{B}_{0}$ in the reference configuration map to positions $\mathbf{y}_{i} \in \mathcal{B}_{t}$ in the deformed configuration at time $t$. Integrals in the peridynamic formulation are naturally discretized as volume-weighted sums over the Voronoi cells. Thus, the deformation gradient is obtained from equation (4) as

$$
\begin{gathered}
\overline{\mathbf{F}}_{j}=\sum_{i=1}^{N_{j}} V_{i} \omega\left(\left|\mathbf{x}_{i}-\mathbf{x}_{j}\right|\right)\left(\mathbf{y}_{i}-\mathbf{y}_{j}\right) \otimes\left(\mathbf{x}_{i}-\mathbf{x}_{j}\right) \overline{\mathbf{K}}_{j}^{-1}, \\
\overline{\mathbf{K}}_{j}=\sum_{i=1}^{N_{j}} V_{i} \omega\left(\left|\mathbf{x}_{i}-\mathbf{x}_{j}\right|\right)\left(\mathbf{x}_{i}-\mathbf{x}_{j}\right) \otimes\left(\mathbf{x}_{i}-\mathbf{x}_{j}\right),
\end{gathered}
$$

where $V_{i}$ is the volume represented by particle $i$, and $i=1, N_{j}$ are the particles in the horizon of particle $j$. The stress and internal state corresponding to this state of deformation is computed for each particle by direct application of the classical constitutive update $\overline{\mathbf{P}}_{j}\left(\overline{\mathbf{F}}_{j}, \mathbf{q}_{j}\right)$. Integrating the force state (5) and replacing in (2) leads to the following semi-discrete equations of motion

$$
\rho \ddot{\mathbf{y}}_{i}=\sum_{j=1}^{N_{i}} V_{j} \omega\left(\left|\mathbf{x}_{j}-\mathbf{x}_{i}\right|\right)\left\{\overline{\mathbf{P}}_{i} \overline{\mathbf{K}}_{i}^{-1}\left(\mathbf{x}_{j}-\mathbf{x}_{i}\right)-\overline{\mathbf{P}}_{j} \overline{\mathbf{K}}_{j}^{-1}\left(\mathbf{x}_{i}-\mathbf{x}_{j}\right)\right\}
$$

These equations of motion are integrated in time using the Newmark time stepping algorithm, with Newmark parameters chosen for explicit time integration and second order accuracy [26], $\beta=0$ and $\gamma=\frac{1}{2}$.

\section{Results}

To assess the functionality of the proposed state-based constitutive correspondence damage formulation, the discretized equations were implemented 
from scratch in a $\mathrm{C}++$ computer code for three dimensional simulations. A Taylor impact test was used to verify our implementation of the state-based peridynamics formulation against other numerical methods for a case involving viscoplasticity but no damage. The proposed peridynamics damage approach was then applied to ballistic impact tests of spherical steel projectiles on extruded aluminum panels.

\subsection{Taylor impact test}

The Taylor impact test [27] has been extensively used for characterizing the dynamic plastic response of materials as well as for validating numerical methods, e.g. $[28,29]$. The test consists of a cylindrical metallic bar impacting a rigid wall at a high velocity. In the case of peridynamics, a Taylor impact test has been used to demonstrate a numerical implementation of the statebased formulation without damage [2]. This test consisted of a $0.0324 \mathrm{~m}$ long 6061-T6 aluminum cylinder with a diameter of $0.00635 \mathrm{~m}$ impacting a rigid wall at $363 \mathrm{~m} / \mathrm{s}$. Instead of using this test, we chose to adopt the specific configuration of the numerical test presented in [29] in order to compare with alternative discretization approaches from the literature $[30,31,32,33]$. In this case, the cylinder material is copper, which is modeled as elastic-plastic with linear isotropic hardening. The cylinder length is $l=0.0324 \mathrm{~m}$ and its radius

$r=0.0032 \mathrm{~m}$. The impact velocity is $227 \mathrm{~m} \cdot \mathrm{s}^{-1}$. The material properties of the cylinder are shown in Table 1. Three different discretizations of 2394 (D1), 16929 (D2) and 130536 (D3) peridynamic particles are used in calculations. Similarly to the approach in [2], for each discretization the horizon size, $\delta$, was chosen to be $3.01 \cdot \Delta x$, where $\Delta x$ is the approximate average distance between neighboring discrete particles.

The final configurations of the bar and contours of equivalent plastic strain are shown in Figure 2. The final radius of the impacting face of the cylinder 


\begin{tabular}{ll} 
Properties & Values \\
\hline Initial density & $8930 \mathrm{~kg} \cdot \mathrm{m}^{-3}$ \\
Bulk modulus & $1.3 \times 10^{11} \mathrm{~N} \cdot \mathrm{m}^{-2}$ \\
Shear modulus & $4.3 \times 10^{10} \mathrm{~N} \cdot \mathrm{m}^{-2}$ \\
Initial yield stress & $4.0 \times 10^{8} \mathrm{~N} \cdot \mathrm{m}^{-2}$ \\
Linear hardening modulus & $1.0 \times 10^{8} \mathrm{~N} \cdot \mathrm{m}^{-2}$
\end{tabular}

Table 1: Material properties used in Taylor impact test simulations.
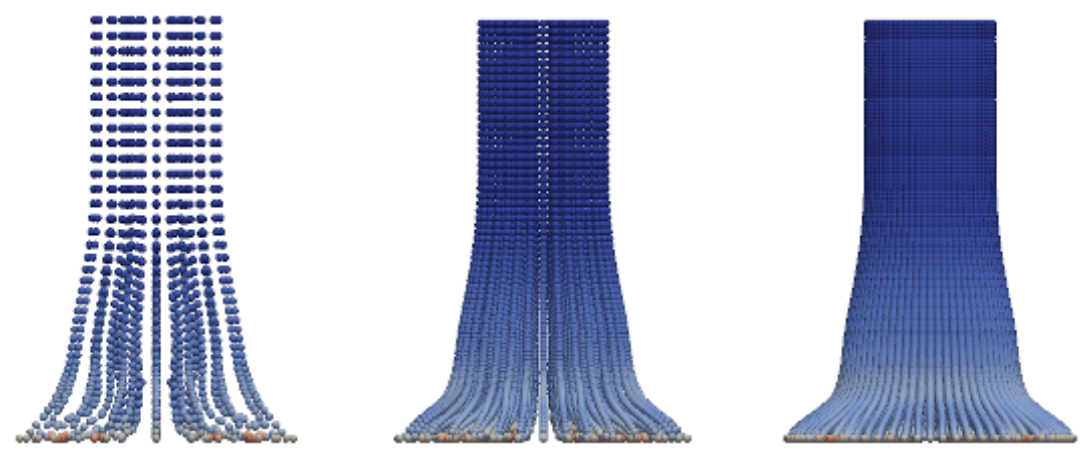

Figure 2: Simulated final configurations of the Taylor impact test for discretizations D1 (left), D2 (center), and D3(right).

$r_{\max }$, the cylinder length $l_{f}$, and the maximum equivalent plastic strain $\epsilon_{\max }^{p}$ are compared to results obtained with finite element calculations [30, 31, 32] and the optimal-transportation method (OTM) [33] in Table 2. With the exception of the results computed by means of the OTM method, the other calculations used for comparison were carried out in axisymmetric mode. It can be seen that the peridynamic simulation results compare reasonably well with results obtained using other numerical methods. These computational results provide an initial verification of the theory, the discretization method, and its computer implementation.

\subsection{Ballistic impact test}

The second example involves the simulation of recent experiments of hardsteel spherical projectiles of diameter 0.55 inches impacting extruded 6061-T6 aluminum sandwich panels [34]. The design of such sandwich structures for im- 


\begin{tabular}{lccc} 
& $l_{f}(\mathrm{~mm})$ & $r_{\max }(\mathrm{mm})$ & $\epsilon_{\max }^{p}$ \\
\hline Kamoulakos [30] & $21.47-21.66$ & $7.02-7.12$ & $2.47-3.24$ \\
Zhu and Cescotto [31] & $21.26-21.49$ & $6.89-7.18$ & $2.47-3.24$ \\
Camacho and Ortiz [32] & $21.42-21.44$ & $7.21-7.24$ & $2.97-3.25$ \\
Li et al. [33] & 21.43 & 6.8 & 3.0 \\
Peridynamics, D1 & 21.5 & 7.1 & 2.69 \\
Peridynamics, D2 & 21.4 & 7.5 & 2.88 \\
Peridynamics, D3 & 21.4 & 7.4 & 3.29 \\
\multicolumn{4}{c}{ Table 2: Comparison of Taylor impact test results. }
\end{tabular}

proved protection performance against impulsive loadings has recently received significant attention $[35,36,37]$. This case provides a stringent test on the ability of the numerical method to model damage, as material damage has a critical influence on the ballistic limit of the structure (the lowest projectile impact velocity for which the target is fully penetrated), and on the residual projectile velocities at higher impact speeds. The left column of Figure 3 shows the post-mortem configurations of the tested panels for different impact velocities.

In simulations, the panel's constitutive response was described by the modified Johnson Cook model, Section 2.3. The Young's modulus E and the strain hardening parameters $n, B$, and $\sigma_{0}$ were calibrated to quasi-static tensile tests, conducted on coupons cut from the faces of the finished panels and measured in the direction of extrusion [38]. Figure 4 shows the quasi-static tensile test stress-strain curve obtained in experiments and the results from the calibrated constitutive model. The parameter $C$ controlling rate-sensitivity in the flow stress was adopted from [39], where it was calibrated to dynamic test data for this material at a reference strain rate $\dot{\epsilon}^{p}=1 \mathrm{~s}^{-1}$. The full set of calibrated constitutive parameter values for the viscoplastic response of the aluminum panels is shown in Table 3. A systematic approach to calibrate damage models for ductile fracture of metals typically involves conducting specific experiments of strain-to-failure for different triaxialities and possibly other parameters charac- 

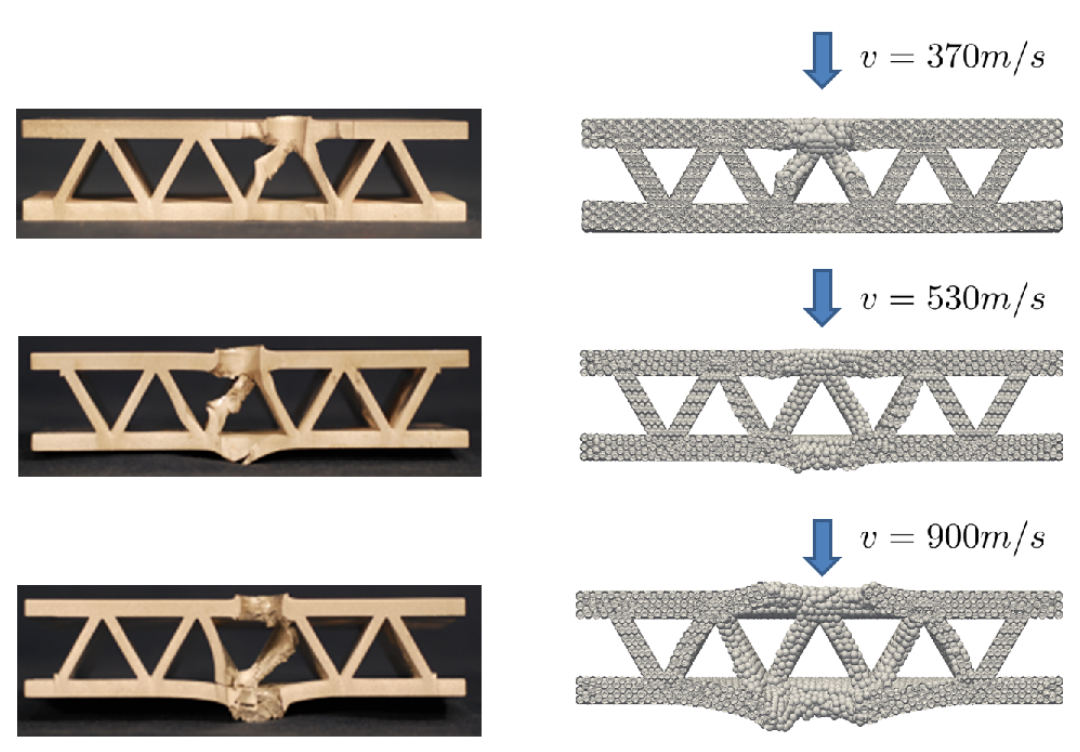

Figure 3: Comparison between numerical simulations and experimental results courtesy of Hayden Wadley, UVA.

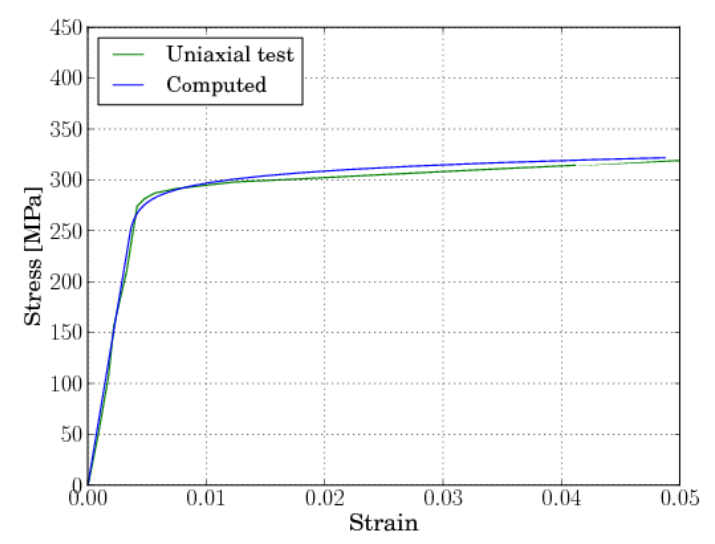

Figure 4: Modified Johnson-Cook material model fit against uniaxial test data [38]. 
terizing the local stress state $[13,14,15]$. However, it is well known that the damage parameters of the model must be recalibrated in simulations depending on the type and size of the discretization [40]. Considering these difficulties and that the emphasis of this paper is in demonstrating the peridynamics description of damage, we calibrated the damage model using a simplified approach where the parameters were adjusted to match a specific measurable metric of a single experiment.

In simulations we represent failure between particles by breaking their bonds when the damage parameter $D_{c}$ in equation (7) exceeds the value 0.99. The choice of a critical damage of 0.99 was chosen arbitrarily and its calibration may have a non-negligible impact on simulation results. Contact between particles is enforced using a simple penalty algorithm which prevents interpenetration of both undamaged and damaged particles. This form of contact is similar to what has been used in the peridynamic literature for particle-on-particle contact [6]. Since the spherical projectiles showed no perceivable plastic deformation in experiments, for simplicity we decided to model them as elastic with density $7800 \mathrm{~kg} / \mathrm{m}^{3}$, Young's modulus $200 \mathrm{GPa}$, and Poisson's ratio 0.25 using a finite element mesh. The boundary conditions for the plate were chosen so as to replicate the experimental conditions by fully constraining the particles along the two long sides of the sandwich specimen. Contact between the finite element mesh (projectile) and the peridynamic domain (sandwich structure) is enforced via penalty contact forces between the nodes of the finite element mesh and the particles in the peridynamic discretization. The peridynamic horizon radius was taken to be approximately three times the largest distance between neighboring particles, which proved to be sufficiently large to ensure reasonably stability of the method.

Simulations were conducted for all of the projectile impact velocities used in 


\begin{tabular}{cccccccc}
$E$ & $\nu$ & $\sigma_{0}$ & $B$ & $n$ & $C$ & $\dot{\epsilon}_{0}^{p}$ & $\rho$ \\
\hline $72 \mathrm{GPa}$ & 0.343 & $250 \mathrm{MPa}$ & $365 \mathrm{MPa}$ & 0.04 & 0.002 & 1 & $2700 \mathrm{~kg} / \mathrm{m}^{3}$
\end{tabular}

Table 3: Material parameters used in numerical simulations.

\begin{tabular}{cccc}
$d_{1}$ & $d_{2}$ & $d_{3}$ & $d_{4}$ \\
\hline 0.875 & 0.13 & -1.5 & 0.0
\end{tabular}

Table 4: Damage parameters used in numerical simulations.

experiments. The simulations were run until the projectile: bounced, partially penetrated and was arrested by the panel, or fully penetrated and exited the back face with a final residual velocity. The Johnson-Cook damage parameters were calibrated using the test-case where the initial projective velocity was $740 \mathrm{~m} / \mathrm{s}$. The parameters, shown in Table 4, were calibrated to exactly reproduce the experimentally observed residual velocity for this test-case. All other simulations were performed using those same parameters.

Figure 3 shows both the experimentally obtained [34] and simulated postmortem deformed configuration of the impacted sandwich structures. The results show that the simulated results accurately predict that an initial projectile velocity of $v=370 \mathrm{~m} / \mathrm{s}$ is well below the ballistic limit. For an initial velocity of $v=530 \mathrm{~m} / \mathrm{s}$, which is near the experimentally predicted ballistic limit, the simulated results accurately predict that the projectile penetrates the top face of the sandwich structure, but is barely stopped by the second layer. At $v=900 \mathrm{~m} / \mathrm{s}$, the prediction that the projectile penetrates both layers is again consistent with the experiments. The numerically computed projectile residual velocity as a function of the initial impact velocity is shown in Figure 5 and is compared with the corresponding experimental results by Wetzel [34]. As it can be observed in this figure, there is a very reasonable agreement between simulations and experiments. 


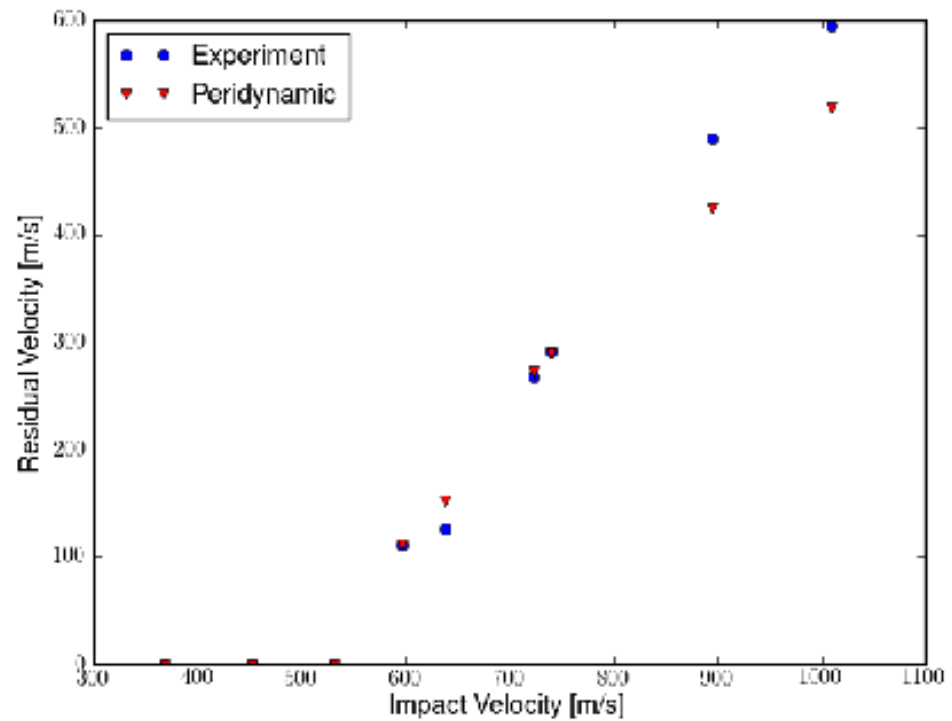

Figure 5: Simulated peridynamic residual velocity vs. initial impact velocity for sandwich panel impact compared to experimental results [34].

\section{Summary and conclusions}

An extension of the peridynamic state-based constitutive correspondence framework to incorporate material damage has been proposed. The main motivation is the desire to incorporate in the modeling framework continuum damage models commonly used in other numerical approaches which have difficulty in simulating problems involving extreme deformations, e.g. the finite element method.

It is found that a direct use of damage models within the constitutive correspondence framework leads to instabilities associated with unphysical diffusion of the damage zone. In order to address this issue, we proposed to progressively degrade the peridynamic influence function appearing in the non-local integral measure of deformation as continuum damage accumulates. This approach effectively generalizes previous approaches which set the influence function to zero 
when a bond extension [11] or a bond elastic energy [10] criterion is satisfied. As a result, fully-damaged material points cease their interaction with their neighbors and damage diffusion issues are avoided.

Benchmark Taylor impact test simulations were performed without damage and compared with established computational approaches, where favorable agreement was found. The method was then tested in the presence of damage for the case of ballistic impact of steel spheres on corrugated aluminum sandwich structures at varying initial velocities. After calibrating the damage evolution law to a single experimental result, both the ballistic limit and the dependence of residual velocity on impact velocity were found to be in close agreement with experimental results. In conclusion, the proposed method furnishes a robust and simple way to incorporate damage models in the peridynamics framework method which is critical for problems involving severe deformations and failure.

\section{Acknowledgements}

The authors acknowledge the support of the Office of Naval Research under Grant N00014-07-1-0764. Partial support from the US Army through the Institute for Soldier Nanotechnologies, under Contract DAAD-19-02-D-0002 with the US Army Research Office is also gratefully acknowledged. The authors are especially grateful to Professor Hayden Wadley and his group at the University of Virginia for their useful input and for providing the experimental data used in the paper.

\section{References}

[1] S.A. Silling, Reformulation of elasticity theory for discontinuities and longrange forces, Journal of the Mechanics and Physics of Solids 48 (2000) 175-209. 
[2] J.T. Foster, S.A. Silling, and W.W. Chen, Viscoplasticity using peridynamics, International Journal for Numerical Methods in Engineering 81 (2010) $1242-1258$.

[3] S.A. Silling and F. Bobaru, Peridynamic modeling of membranes and fibers, International Journal of Non-Linear Mechanics 40 (2004) 395-409.

[4] S.A. Silling and E. Askari, A meshfree method based on the peridynamic model of solid mechanics, Computers and Structures 83 (2005) 1526-1535.

[5] E. Askari, F. Bobaru, R.B. Lehoucq, M.L. Parks, S.A. Silling, and O. Weckner, Peridynamics for multiscale materials modeling, Journal of Physics: Conference Series 125 (2008) 012078.

[6] M.L. Parks, R.B. Lehoucq, S. Plimpton, and S. Silling, Implementing peridynamics within a molecular dynamics code, Computer Physics Communications 179 (2008) 777-783.

[7] S.A. Silling, O. Weckner, E. Askari, and F. Bobaru, Crack nucleation in a peridynamic solid, International Journal of Fracture 162 (2010) 219-227.

[8] Y.D. Ha and F. Bobaru, Studies of dynamic crack propagation and crack branching with peridynamics, International Journal of Fracture 162 (2010) $229-244$.

[9] S.A. Silling, M. Epton, O. Weckner, J. Xu, and E. Askari, Peridynamic states and constitutive modeling, Journal of Elasticity 88 (2007) 151-184.

[10] J.T. Foster, Dynamic Crack Initiation Toughness: Experiments and Peridynamic Modeling, Technical Report SAND2009-7217, Sandia National Laboratories, Albuquerque. 
[11] P. Seleson, Peridynamic multiscale models for the mechanics of materials: constitutive relations, upscaling from atomistic systems, and interface problems, Ph.D. thesis, Florida State University (2010).

[12] P. Seleson and M.L. Parks, On the role of the influence function in the peridynamic theory, Journal for Multiscale Computational Engineering 9 (6) (2011) 689-706.

[13] Y. Bao, T. Wierzbicki, A comparative study on various ductile crack formation criteria, Journal of Engineering Materials and Technology 126 (2004) $314-324$.

[14] K. Nahshon and J.W. Hutchinson, Modification of the Gurson model for shear failure, European Journal of Mechanics A/Solids 27 (2008) 1-17.

[15] Y. Bai, T. Wierzbicki, Application of extended Mohr-Coulomb criterion to ductile fracture, International Journal of Fracture 161 (2010) 1-20.

[16] S.A. Silling, and R.B. Lehoucq, Peridynamic theory of solid mechanics, Advances in Applied Mechanics 44 (2010) 73-168.

[17] G.R. Johnson and W.H. Cook, Fracture characteristics of three metals subjected to various strains, strain rates, temperatures and pressures, Engineering Fracture Mechanics 21 (1985) 31-48.

[18] D.L. Littlewood, Simulation of dynamic fracture using peridynamics, finite element modeling, and contact, Proceedings of the ASME 2010 International Mechanical Engineering Congress \& Exposition.

[19] S. Beissela and T. Belytschko, Nodal integration of the element-free galerkin method, Computer Methods in Applied Mechanics and Engineering 139 (1996) 49-74. 
[20] T. Belytschko and Y. Guox and W. K. Liu and S. P. Xia, A unifed stability analysis of meshless particle methods, International Journal for Numerical Methods in Engineering 48 (2000) 1359-1400.

[21] B. Kilic and E. Madenci, Prediction of crack paths in a quenched glass plate using peridynamic theory, International Journal of Fracture 156 (2009) $165-177$.

[22] G.R. Johnson and W.H. Cook, A constitutive model and data for metals subjected to large strains, high strain rates and high temperatures, in: Proceedings of the 7th International Symposium on Ballistics, The Hague, Netherlands, 1983, pp. 541-547.

[23] R. Radovitzky and M. Ortiz, Error estimation and adaptive meshing in strongly nonlinear dynamic problems, Computer Methods in Applied Mechanics and Engineering 172 (1999) 203-240.

[24] M. Ortiz, L. Stainier, The variational formulation of viscoplastic updates, Computer Methods in Applied Mechanics and Engineering 171 (1999) 419444.

[25] Q. Yang, L. Stainier, M. Ortiz, A variational formulation of the coupled thermo-mechanical boundary-value problem for general dissipative solids, Journal of the Mechanics and Physics of Solids 54 (2006) 401-424.

[26] T. Belytschko and T.J.R. Hughes, Computational Methods for Transient Analysis, Elsevier Science, North-Holland, 1983.

[27] G.I. Taylor, The use of flat-ended projectiles for determining dynamic yield stress i. theoretical considerations, Proceedings of the Royal Society of London. Series A. Mathematical and Physical Sciences 194 (1948) 289-299. 
[28] G. R. Johnson, T. J. Holmquist, Evaluation of cylinder-impact test data for constitutive models, Journal of Applied Physics 64 (8) (1988) 3901-3910.

[29] J.C. Simo, Algorithms for static dynamic multiplicative plasticity that preserve the classical return mapping schemes of the infinitesimal theory, Computer Methods in Applied Mechanics and Engineering 99 (1992) 61-112.

[30] A. Kamoulakos, A simple benchmark for impact, Benchmark (1990) 31-35.

[31] Y.Y. Zhu and S. Cescotto, Unified and mixed formulation of the 4-node quadrilateral elements by assumed strain method: application to thermomechanical problems, International Journal for Numerical Methods in Engineering 38 (1995) 685-716.

[32] G.T. Camacho and M. Ortiz, Adaptive lagrangian modelling of ballistic penetration of metallic targets, Computer Methods in Applied Mechanics and Engineering 142 (1997) 269-301.

[33] B. Li, F. Habbal, and M. Ortiz, Optimal transportation meshfree approximation schemes for fluid and plastic flows, International Journal for $\mathrm{Nu}-$ merical Methods in Engineering 83 (2010) 1541-1579.

[34] J.J. Wetzel, The impulse response of extruded corrugated core aluminum sandwich structures, Master's thesis, University of Virginia (2009).

[35] L.F. Mori, S. Lee, Z.Y. Xue, A. Vaziri, D.T. Queheillalt, K.P. Dharmasena, H.N.G. Wadley, J.W. Hutchinson and H.D. Espinosa, Deformation and fracture modes of sandwich structures subjected to underwater impulsive loads, Journal of Mechanics of Materials and Structures 2 (2007) 19812006.

[36] H.N.G. Wadley, Multifunctional periodic cellular metals, Philisophical Transactions of the Royal Society A 364 (2006) 31-68. 
[37] T. Rabczuk, E. Samaniego, and T. Belytschko, Simplified model for predicting impulse loads on submerged structures to account for fluid-structure interation, International Journal of Impact Engineering 34 (2007) 163-177.

[38] J.J. Rimoli, B.Talamini, J.J. Wetzel, K.P. Dharmasena, R. Radovitzky and H.N.G. Wadley, Wet-sand impulse loading of metallic plates and corrugated core sandwich panels, International Journal of Impact Engineering 38 (2011) 837-848.

[39] K. Dannemann, C. Anderson, G. Johnson, Modeling the ballistic impact performance of two aluminum alloys, in: D. Lesuer, T. Srivatsan (Eds.), Proceedings of the symposium modeling the performance of engineering structural materials II, The Minerals, Metals and Materials Society (TMS), Indianapolis, Indiana, USA, 2001, pp. 63-74.

[40] Committee on Opportunities in Protection Materials Science and Technology for Future Army Applications; National Research Council, Opportunities in Protection Materials Science and Technology for Future Army Applications, National Academies Press, 2011. 